\title{
Estrategias de afrontamiento al estrés y síntomas patológicos en universitarios ante un desastre socionatural de aluvión de barro
}

\author{
Jorge Alejandro Salgado Roa* y Francisco José Leria Dulčić \\ Departamento de Psicología, Instituto de Investigación en Ciencias Sociales y Educación, Universidad de Atacama, Copiapó, Chile
}

Recibido, mayo 5/2017

Concepto de evaluación, julio 4/2017

Aceptado, agosto 11/2017
Referencia: Salgado Roa, J.A. \& Leria Dulcic, F.J. (2018). Estrategias de afrontamiento al estrés y síntomas patológicos en universitarios ante un desastre socionatural de aluvión de barro. Acta colombiana de Psicología, 21(1), 170-182. doi: http://www.dx.doi.org/10.14718/ACP.2018.21.1.8

\section{Resumen}

En la presente investigación se analiza la asociación entre sintomatología psicopatológica, estrategias de afrontamiento al estrés y variables sociodemográficas relacionadas con la vivencia de un desastre socionatural. En total, participaron 399 universitarios que completaron las escalas de los síntomas psicológicos (SCL-90-R), estrategias de afrontamientos (CSI) y una encuesta sociodemográfica. Se utilizó una estrategia asociativa-comparativa transversal y un diseño de grupos naturales. Los resultados indicaron la existencia de diferencias debidas al sexo en los síntomas psicopatológicos de somatización, depresión, y ansiedad, siendo las mujeres quienes puntuaron más alto. Se presentan diferencias con respecto a la valoración global del impacto producido por un aluvión en todas las dimensiones psicopatológicas, excepto en sensibilidad interpersonal; y los análisis de regresión múltiple mostraron que las dimensiones psicopatológicas son explicadas por alta retirada social y pensamiento desiderativo. Estos resultados sugieren que tanto las estrategias de afrontamiento al estrés como la presencia de niños en el núcleo familiar, la exposición a la violencia, el deterioro de la vivienda y la valoración de impacto, son potentes predictores de la sintomatología de la depresión, el índice sintomático general, la ansiedad, la somatización, la obsesióncompulsión y el total de síntomas positivos.

Palabras clave: Síntomas patológicos, estrategias de afrontamiento, psicología de la emergencia, desastres socionaturales.

\section{Coping strategies for stress and pathological symptoms in university students in the face of a mudslide disaster}

\begin{abstract}
This paper aimed to analyze the association between psychopathological symptomatology, stress coping strategies and sociodemographic variables related to the experience of social disaster. 399 undergraduates completed the Psychological Symptom Scales (SCL-90-R), the Coping Strategies Inventory (CSI) and a sociodemographic survey. A cross-sectional associative-comparative strategy and a natural group design were used. The results indicated the existence of differences due to sex in the psychopathological symptoms of Somatization, Depression, and Anxiety, where women scored higher. There are differences regarding the global assessment of the impact caused by the flood in all psychopathological dimensions, except for Interpersonal Sensitivity. Multiple regression analyzes showed that the psychopathological dimensions are explained by high Social Withdrawal and Desiderative Thinking. These results suggest that not only stress coping strategies, but the presence of children in the family nucleus, exposure to violence, housing deterioration and impact assessment are potent predictors of symptoms of Depression, General Symptomatic Index, Anxiety, Somatization, Obsession-Compulsion, and Total Positive Symptoms.

Key words: Pathological symptoms, coping strategies, emergency psychology, socio-natural disasters.
\end{abstract}

* Copayapu 485, Copiapó, Chile; Tel.:56+966594419; jorge.salgado@uda.cl

** Copayapu 485, Copiapó, Chile; Tel.:56+981997981; francisco.leria@uda.cl

Se agradece a los Académicos del Departamento de Psicología de la Universidad de Atacama (Chile) por colaborar activamente en el proceso de levantamiento de datos. De igual forma, se agradece a la académica del Departamento de Idiomas de la misma institución, la Srta. Gisella Naranjo Saavedra por realizar la traducción del presente artículo. 


\title{
Estratégias de enfrentamento do estresse e sintomas patológicos em universitários ante um desastre socionatural de deslizamento de terra
}

\author{
Resumo
}

\begin{abstract}
Na presente pesquisa, analisa-se a associação entre sintomatologia psicopatológica, estratégias de enfrentamento do estresse e variáveis sociodemográficas relacionadas com a vivência de um desastre socionatural. Em total, participaram 399 universitários que completaram as escalas dos sintomas psicológicos (SCL-90-R), estratégias de enfrentamentos (CSI) e um questionário sociodemográfico. Utilizou-se estratégia associativa-comparativa transversal e desenho de grupos naturais. Os resultados indicaram a existência de diferenças devido ao sexo nos sintomas psicopatológicos de somatização, depressão e ansiedade, sendo as mulheres as que pontuaram mais alto. Apresentam-se diferenças a respeito da valoração global do impacto produzido por um deslizamento de terra em todas as dimensões psicopatológicas, exceto em sensibilidade interpessoal; as análises de regressão múltipla mostraram que as dimensões psicopatológicas são explicadas por alta retirada social e pensamento desiderativo. Esses resultados sugerem que tanto as estratégias de enfrentamento do estresse quanto a presença de crianças no núcleo familiar, a exposição à violência, a deterioração da moradia e a valoração de impacto são potentes preditores da sintomatologia da depressão, do índice sintomático geral, da ansiedade, da somatização, da obsessão-compulsão e do total de sintomas positivos.

Palavras-chave: Desastres socionaturais, estratégias de enfrentamento, psicologia da emergência, sintomas patológicos.
\end{abstract}

\section{INTRODUCCIÓN}

La calidad adaptativa de la respuesta psicológica de un sujeto ante una situación de desastre socionatural es modulada por su capacidad de afrontamiento ante el mismo. Las denominadas estrategias de afrontamiento -0 coping skills-(en adelante, EA), se definen como aquellos esfuerzos cognitivos, emocionales y conductuales para manejar las demandas específicas, externas o internas evaluadas como excesivas o que sobrepasan de los recursos del sujeto(Lazarus \& Folkman, 1986); y se constituyen como estrategias psicosociales frente al estrés tanto en contextos ocupacionales como situacionales varios (Loo et al., 2016). La evidencia actual categoriza a las EA dependiendo de si están focalizadas al problema o centradas en la gestión de la emoción; si son primarias (asimilativas) o secundarias (acomodativas); o si son de acercamiento o de evitación (Pfefferbaum et al., 2016).

Teóricamente, se afirma que las condiciones que llevan al aparato cognitivo a construir EA exitosas se relacionan con la presencia de entornos con un bajo grado de incertidumbre, los cuales le permiten al sujeto anticipar soluciones exitosas frente a problemas actuales. No obstante, situaciones inesperadas o extremas, como en el caso de los desastres socionaturales, generan respuestas desadaptativas (Leiva-Bianchi, 2011), pues frente a tales condiciones el sujeto tiende a ver debilitadas sus capacidades, además de que recibe un impacto global en varias de sus funciones psicológicas, lo que resulta en un deterioro cognitivo y una mayor probabilidad para el desarrollo de trastornos depresivos, ansiosos o de identidad, y, en algunos casos, desordenes psiquiátricos como el trastorno por estrés postraumático (TEPT) (Leach, 2016).
Al respecto, la investigación psicosocial en desastres ha abordado el estudio del comportamiento adaptativo ante eventos desastrosos, en los cuales las personas se ven desbordadas en los mecanismos habituales de afrontamiento (López, Christodoulou, Maj, Sartorius \& Okasha, 2005); y se ha caracterizado por una importante proliferación de estudios en la actualidad con respecto a algunos conceptos asociados, como la vulnerabilidad, las EA y la gestión del riesgo (Aledi \& Sulaiman, 2014).

Por ejemplo, la evidencia empírica constata diferencias en las EA al estrés de acuerdo con diversas variables constitutivas y circunstanciales del sujeto, como el ciclo vital, por ejemplo (Novais, Monteiro, Roque, Correia-Neves \& Sousa, 2017). Específicamente, estudios meta-analíticos concluyen que las EA más utilizadas por la población joven (adolescentes y adultos jóvenes) se agrupan en los ámbitos: (1) sociocultural, (2) escolar, (3) familiar y amigos, y (4) sí mismo; y funcionalmente sobresalen las EA de (a) carácter positivo -como elaboración de un plan, optimismo, religión/ espiritualidad, búsqueda de apoyo social y humor-; y (b) de carácter negativo -como distanciamiento/desvinculación, rumiación mental, ira, perfeccionismo, abuso de substancias, pensamiento negativo y huida en reflexiones religiosas evitativas- (Montgomery et al., 2014).

Por otra parte, diferentes estudios demuestran que las EA preferidas por la población infantil se circunscriben al uso de las emociones (Mestre, Samper, Tur-Porca, de Minzi \& Mesurado, 2012); y en la población escolar a la solución activa, la búsqueda de información, la emoción y apoyo social, la ocultación del problema y la pasividad (Morales, Trianes, Miranda \& Inglés, 2016). En particular, los niños y los adolescentes se encuentran entre las poblaciones con mayor 
vulnerabilidad al impacto de las catástrofes (Powel \& Bui, 2016), pues, debido a un importante correlato neurofisiológico, en sujetos entre los 15 y 17 años se manifiestan altos niveles de secreción de cortisol por el aumento funcional del eje hipotalámico-pituitario-adrenal (HPA) y el eje hipotalámicopituitario-gonadal (HPG), incluso mayores que en la infancia tardía o pubertad temprana (Gunnar, Wewerka, Frenn, Long \& Griggs, 2009; Ortiz, Silverman, Jaccard \& La Greca, 2011). Adicionalmente, la exposición a un estrés intenso podría inducir cambios estructurales en la expresión hormonal de los receptores del encéfalo y, por tanto, en la respuesta conductual ante futuras situaciones de estrés (Blaustein \& Ismail, 2013).

Teniendo esto en cuenta, la prevalencia de la sintomatología asociada a la exposición a un desastre socionatural se encuentra en un rango extremadamente variable, que va de un $4 \%$ hasta un exorbitante $70 \%$; de $4.4 \%$ a $36 \%$ en Chile (Pérez et al., 2009); y, en el caso de jóvenes que directamente experimentaron una reacción postraumática ante un desastre, la evidencia sugiere que el $27 \%$ mantiene su sintomatología durante tres meses después del evento (Neria, Nandi \& Galea, 2008), y hasta después de dos años (Roberts, Mitchell, Witman \& Taffaro, 2010), sintomatología relacionada con la victimización, la depresión, la ansiedad, los comportamientos agresivos (Becker, Turner \& Finkelhor, 2010) y el sentimiento de culpa (Salloum \& Overstreet, 2012).

Asimismo, estudios contemporáneos han enfatizado el empeoramiento de las EA ante situaciones de desastres en la población adolescente (Daxing, Huifang, Shujing \& Ying, 2011), sobre todo por la falta de control y la pérdida de confianza (Pineda \& López, 2010), o por la escogencia de estrategias como la evitación, la negación y el pensamiento desiderativo, que tienen poco efecto e incluso exacerban los síntomas de TEPT a lo largo del tiempo (Wadsworth, Raviv, Compas \& Connor-Smith, 2005).

Sin embargo, así como se han encontrado estrategias negativas, también se han detectado EA que poseen un factor protector que le otorga al sujeto una mayor autoeficacia en la solución de los problemas relacionados con la situación de estrés experimentada, tales como la inteligencia, las estrategias autorregulativas, la esperanza y significados transcendentes de la vida, la autoeficacia, las relaciones de apoyo social, las creencias y prácticas religiosas, el apoyo comunitario (Powell \& Bui, 2016), la independencia social, la iniciativa interpersonal, la responsabilidad y apertura social (Ling-Xiang \& Cody, 2011), la estabilidad emocional percibida (Hussain, Weisæth \& Heir, 2013) y la condición física (Momma et al., 2014). Y, en línea con estos hallazgos, la EA de resiliencia ha explicado el $70 \%$ de la respuesta moduladora para la aparición del TEPT y la ideación suicida, actuando como un amortiguador de los estresores (Stratta et al., 2015).
Por otra parte, existe una especial preocupación por la alta prevalencia del TEPT en adultos jóvenes víctimas de aluviones, pues se ha encontrado una prevalencia de $25.8 \%$ y alta presencia de sintomatología ansiosa de tipo irruptora e intrusiva en el desarrollo cognitivo (Pinchen et al., 2011), así como depresión y ansiedad generalizada (Lima, Santacruz, Lozano, Luna \& Pai, 1988), hiperactivación autonómica, pensamientos intrusivos, hiperactivación psicológica y evitación (Craparo, Faraci, Rotondo \& Gori, 2013).

Ahora bien, es importante recordar que el 25 de marzo del 2015 se precipitó un desastre pluviométrico que desbordó ríos y deslizó tierras provenientes de relaves mineros $\left(27^{\circ} 21^{\prime} 59^{\prime \prime} \mathrm{S}\right.$, $70^{\circ} 19^{\prime} 59^{\prime \prime} \mathrm{W}$ ) en las regiones chilenas de Antofagasta, Atacama y Coquimbo. El gobierno decretó zona de catástrofe y luego excepción constitucional, razón por la cual militares se hicieron cargo del resguardo del orden público. Posteriormente, la población fue expuesta a una serie de eventos estresantes consecuentes a la catástrofe, sobre todo por la contaminación del aire, los daños en las vías públicas y peatonales, y la basura y montones de barro apilados, entre otros efectos y residuos del aluvión. Los datos oficiales cuentan más de 28.000 damnificados, 31 personas fallecidas y 16 desaparecidas (Oficina Nacional de Emergencia del Ministerio del Interior [ONEMI], 2015). Además, se estableció la existencia de un $43 \%$ de viviendas con daños reparables, un $23 \%$ con daños leves, un $13 \%$ con daños moderados, un $7 \%$ con daños mayores, y de un $6 \%$ con daños no reparables (Ministerio de Vivienda y Urbanismo [MINVU], 2015).

En esta zona, tras la ocurrencia del desastre que inspira esta investigación, un estudio preliminar muestra que en estudiantes universitarios se encontró un $2 \%$ de síntomas de TEPT y un $85 \%$ de síntomas de mediana intensidad ante el impacto del evento, lo que sugiere, a pesar de la baja prevalencia de TEPT, una alta presencia de estrés subjetivo (Lería \& Salgado, 2016).

A partir de lo expuesto se desprende la necesidad de incurrir en estudios que den cuenta de las estrategias moduladoras de la respuesta sintomatológica ante tales situaciones. Para esto, las preguntas que guían el presente estudio son: (1) ¿existen diferencias entre los síntomas psicopatológicos y las EAal estrés en función de variables sociodemográficas y relacionadas con la vivencia del aluvión?,(2) ¿cuál es la relación entre síntomas psicopatológicos y las EA al estrés?, y (3) ¿qué variables explican significativamente la variabilidad de la sintomatología psicopatológica? De esta forma, se desprende como objetivo del estudio identificar la asociación entre la sintomatología psicopatológica, las EA y las variables sociodemográficas relacionadas con la vivencia del desastre socionatural de aluvión de barro en una ciudad de tamaño mediano. 


\section{MÉTODO}

\section{Diseño}

El presente estudio adopta un diseño no experimental transeccional ex post facto con finalidad descriptiva. Es decir que no se manipularon variables, sino que se analizaron las relaciones entre estas y se indagó sobre las diferencias entre dos o más grupos de individuos a partir de los contrastes generados por la naturaleza y la sociedad (Ato, López \& Benavente, 2013).

\section{Participantes}

Se utilizó un muestreo de tipo aleatorio estratificado, donde se calculó un margen de error del $5 \%$ y un nivel de confianza del $99 \%$, y se obtuvo una muestra de 408 estudiantes de un total de 1157 alumnos de una universidad pública, pertenecientes a cuatro facultades académicas (Ingeniería, Humanidades y Educación, Ciencias Jurídicas y Sociales, y Ciencias de la Salud), las cuales se constituyeron en los estratos de donde fueron seleccionados aleatoriamente los universitarios (Amón, 1995).

Es importante señalar que nueve participantes fueron descartados del análisis por no completar todos los instrumentos, de manera que la población se redujo a 399 estudiantes (275 mujeres y 124 hombres), con edades comprendidas entre los 18 y 64 años $(M=20.9, D E=4.26)$, de los cuales el $53.6 \%$ cursaba primer nivel, el $28.6 \%$ el segundo nivel, el $5.8 \%$ el tercer nivel, y el $8.3 \%$ el cuarto nivel educativo (año que cursa en la universidad). Respecto del estado civil, el $94 \%$ de los estudiantes reportó ser soltero y el $2 \%$ dijo que estaba casado. Además, el $64.4 \%$ informó de la presencia de niños en su núcleo familiar, mientras que el $35.3 \%$ reportó no contar con dichos miembros en sus hogares.

\section{Instrumentos}

Teniendo en cuenta las sugerencias de Balaban (2006) con respecto a la investigación en víctimas de desastres socionaturales, los participantes completaron los siguientes cuestionarios, en una aplicación aproximada de 60 minutos:

Cuestionario de sintomas psicopatológicos revisado (SCL-90-R) (Derogatis, 2002). En la adaptación española de González, De las Cuevas, Rodríguez y Rodríguez (2002), es un cuestionario autoaplicado que permite evaluar nueve dimensiones psicológicas (somatización, obsesión-compulsión, sensibilidad interpersonal, depresión, ansiedad, hostilidad, ansiedad fóbica, ideación paranoide y psicoticismo), a partir de 90 reactivos con escalamiento tipo Likert de cinco categorías, que va desde 0 (nada en absoluto) hasta 4 (mucho o extremadamente).

Este instrumento permite obtener tres índices generales: el índice global de gravedad (GSI), el total de síntomas positivos (PST) y el índice de distrés de síntomas positivos (PSDI) (Rosa-Alcázar, Parada-Navas \& Rosa-Alcázar, 2014); y puede ser utilizado tanto en pacientes en tratamiento psicológico o psiquiátrico como en población general (Sánchez \& Ledezma, 2009). Los valores tanto de los coeficientes de confiabilidad como los de estabilidad temporal se encuentran entre .78 y .90 , con un intervalo de test-retest de una semana, y presentan estabilidad de las puntuaciones a lo largo del tiempo (Derogatis, 2002). En el presente estudio, los coeficientes de confiabilidad alfa de Cronbach se encuentran entre .71 y .89, a excepción de la variable ideación paranoide, que presenta un alfa de 687 .

Inventario de estrategias de afrontamiento (CSI) (Tobin, Holroyd, Reynolds \& Kigal, 1989). En la versión española de Cano, Rodríguez y García, (2007), es una escala utilizada para determinar las EA, compuesta por 40 reactivos con escalamiento tipo likert de cinco puntos, que va de 0 (en absoluto) hasta 4 (totalmente). Al final de la prueba se responde a un ítem adicional acerca de la autoeficacia percibida del afrontamiento.

Este instrumento permite evaluar ocho escalas primarias (resolución de problemas, autocrítica, expresión emocional, pensamiento desiderativo, apoyo social, reestructuración cognitiva, evitación de problemas y retirada social), y presenta coeficientes de fiabilidad alfa de Cronbach altos (entre .78 y .89), a excepción de evitación de problemas $(\alpha=.63)$ y retirada social $(\alpha=.65)$ (Cano et al., 2007); valores similares a los obtenidos en la presente investigación (alfas entre .74 y.82), a excepción de autocrítica $(\alpha=.65)$, evitación de problemas $(\alpha=.68)$, y retirada social $(\alpha=.68)$.

Encuesta sociodemográfica. Esta encuesta fue elaborada como estrategia ad hoc para recolectar información referente a variables cuantitativas y cualitativas de tipo sociodemográfico y relacionadas con la percepción sobre la experiencia de vida durante y después al aluvión de barro, como, por ejemplo: dirección al momento del aluvión; valoración del impacto global del aluvión; existencia de niños, ancianos o personas con discapacidad en el núcleo familiar; deterioro, perdida o destrucción de la vivienda; exposición a robos o violencia; alteración de la situación laboral o pérdida de ingresos, entre otras. 


\section{Procedimiento}

Este trabajo contó con la autorización de las autoridades de la institución participante, y el procedimiento utilizado fue el siguiente: (a) se expusieron los objetivos del estudio a los decanos de las distintas facultades académicas y a los directores de las carreras colaboradoras; (b) se hizo la selección de una muestra representativa por carrera, nivel y sexo; (c) se realizó la coordinación con los directores para determinar el lugar de aplicación de los instrumentos; (d) se informó a los participantes de la finalidad de la investigación y del resguardo de la confidencialidad de la información, además de que se recalcó el carácter voluntario y la posibilidad de interrumpir la participación en el proceso; y (e) se procedió a la lectura del consentimiento informado y a la aplicación anónima de los instrumentos, la cual se realizó de forma grupal, por carrera y nivel cursado.

\section{Análisis de datos}

Para describir las dimensiones psicológicas y las escalas generales del SCL-90-R y las EA, se calcularon los promedios y desviaciones estándar respectivos, así como las correlaciones lineales de Pearson $(r)$ entre las puntuaciones directas obtenidas con ambos instrumentos, mientras que para determinar el uso de pruebas paramétricas se corroboró el cumplimiento de los supuestos de normalidad, con el test de Kolmogorov-Smirnov (K-S) y la corrección de Lilliefors, y el de homocedasticidad, con el uso de la prueba de Levene, (Amón, 1996).

Posteriormente, se analizaron los síntomas psicopatológicos y las dimensiones de afrontamiento en función de las variables sociodemográficas y aquellas relacionadas con la percepción sobre la experiencia de vida durante y posterior al aluvión de barro. Para las diferencias según el sexo, se utilizó la prueba de $t$ de Student para muestras independientes. En el caso de no existir homogeneidad, se aplicaría el test de Welch (Armitaje, Berry \& Matthews, 1994), pero para el resto de las variables se realizó un análisis de varianza unifactorial ANOVA.

Las comparaciones post hoc se realizaron aplicando la prueba de Scheffé, y para determinar el tamaño del efecto (TE) se calculó la $d$ de Cohen $(d)$ y el eta cuadrado parcial $\left(\eta_{p}^{2}\right)$ (Cohen, 1988). Finalmente, se ingresó a un modelo de regresión lineal múltiple, y con el método por pasos (stepwise) se examinó la capacidad predictiva de la experiencia percibida en torno al desastre socionatural y las EA al estrés sobre las dimensiones psicopatológicas. Para el procesamiento de los datos se utilizó el software SPSS 22.0.

\section{Consideraciones éticas}

Cabe destacar que el presente estudio fue revisado y aprobado por el Comité de Ética Científica de la institución de los autores. Los estudiantes no recibieron ningún incentivo por participar y se tomaron todos los resguardos para garantizar el cumplimiento de los aspectos éticos de la investigación con seres humanos propuestos en las directrices de la American Psychological Association.

\section{RESULTADOS}

En el siguiente apartado se presentan los resultados del análisis de los datos comenzando con el examen comparativo de los síntomas patológicos y las EA en cuanto a las variables independientes del estudio. Posteriormente, se muestran las correlaciones de las puntuaciones directas obtenidas en los instrumentos aplicados, y, finalmente, se muestran los resultados del modelo de regresión por pasos en la sintomatología psicopatológica.

Diferencias en los sintomas psicopatológicos y EA al estrés en función de variables sociodemográficas y relacionadas con la vivencia del aluvión.

En la Tabla 1 se observa la existencia de diferencias en función del sexo en los síntomas psicopatológicos de somatización $(p=.001)$, depresión $(p=.010)$, y ansiedad ( $p=.033)$, siendo los valores medios de las mujeres más altos que los de los hombres. Considerando los TE, es posible señalar que las diferencias son pequeñas, según la interpretación de Cohen (1988), y las puntuaciones obtenidas indican que la muestra se encuentra entre los percentiles 55 al 90, destacando las medias de ambos sexos en obsesióncompulsión y depresión.

Con respecto a las EA al estrés, se encontraron diferencias en función del sexo en expresión emocional $(p=.014)$, pensamiento desiderativo ( $p=.002$ ), pues en estas se encontraron medias más elevadas en las mujeres; y en evitación de problemas $(p=.018)$, que obtuvo un mayor puntaje en los hombres. Acá, los TE fueron pequeños, y cabe destacar que los participantes de ambos sexos presentaron medias elevadas en pensamiento desiderativo, reestructuración cognitiva, evitación de problemas y retirada social.

Por otra parte, en las correlaciones entre edad y sintomatología psicopatológica no se obtuvieron relaciones significativas, pero entre edad y EA al estrés se encontraron correlaciones significativas y directas en las variables autocrítica $\left(r_{(389)}=.101, p=.047\right)$, expresión emocional $\left(r_{(389)}=.123\right.$, $p=.016)$ y reestructuración cognitiva $\left(r_{(389)}=.108, p=.034\right)$. 
Tabla 1

Diferencias en las dimensiones del SCL-90-R y el CSI en función del sexo

\begin{tabular}{lccccc}
\hline \multicolumn{1}{c}{ Escala/Dimensión } & \multicolumn{2}{c}{ Media (DE) } & \multirow{2}{*}{$(397)$} & $\mathrm{p}$ & $\mathrm{d} \mathrm{de}$ \\
Cohen
\end{tabular}

Nota. *Las varianzas son desiguales, por lo que se aplica el test de Welch.

En cuanto al lugar donde habitaban los participantes, según el grado de afectación en tres zonas (Amarillo $=n o$ afectado, Verde $=$ medianamente afectado y Rojo $=$ muy afectado) (MINVU, 2015), se encontraron diferencias significativas en las medias de todos los síntomas psicopatológicos -incluyendo los índices globales-a excepción de ansiedad fóbica $\left(F_{(2.374)}=2.202, p=.112\right)$. Mientras que con respecto a las EA al estrés se presentan medias desiguales en resolución de problemas $\left(F_{(3.373)}=3.439, p<.05\right) \mathrm{y}$ pensamiento desiderativo $\left(F_{(3.374)}=3.135, p<.05\right)$. Cabe destacar que las puntuaciones medias más altas, en todos los casos, corresponden a los participantes que habitan la zona de mayor afectación.

Adicionalmente, respecto al nivel que cursa el estudiante, no se encontraron diferencias significativas en los síntomas psicopatológicos; mientras que en las EA al estrés se encontró lo contrario, especialmente en las variables autocritica $\left(F_{(3.380)}=2.683, p<.05, \eta_{\mathrm{p}}{ }^{2}=.024\right)$, expresión emocional $\left(F_{(3.380)}=4.631, p<.01, \eta_{\mathrm{p}}{ }^{2}=.033\right)$, apoyo so$\operatorname{cial}\left(F_{(3.380)}=6.018, p<.045, \eta_{\mathrm{p}}{ }^{2=} .045\right)$, reestructuración cognitiva $\left(F_{(3.380)}=5.015, p<.01, \eta_{\mathrm{p}}{ }^{2=} .032\right)$ y evitación de problemas, $\left(F_{(3.380)}=2.702, p<.05, \eta_{\mathrm{p}}{ }^{2}=.019\right)$. Cabe destacar que las medias más altas se presentaron en los niveles superiores (tercer y cuarto nivel), y que los TE fueron pequeños según la clasificación de Cohen (1988).

En cuanto al estado civil de los participantes no se encontraron diferencias significativas en la sintomatología psicopatológica ni en las EA al estrés. Pero en el tipo de carrera cursada se encontraron diferencias significativas en la sintomatología, por ejemplo, en somatización $\left(F_{(14.371)}=3.583, p<.001, \eta_{\mathrm{p}}{ }^{2}=.135\right)$, obsesión-compulsión $\left(F_{(14.371)}=4.083, p<.001, \eta_{\mathrm{p}}^{2}=.159\right)$, sensibilidad interpersonal $\left(F_{(14.371)}=2.055, p<.05, \eta_{\mathrm{p}}{ }^{2}=.087\right)$, depresión $\left(F_{(14.371)}=2.561, p<.01, \eta_{\mathrm{p}}{ }^{2}=.102\right)$, ansiedad $\left(F_{(14.371)}=2.395\right.$, $\left.p<.05, \eta_{\mathrm{p}}{ }^{2}=.092\right)$, ansiedad fóbica $\left(F_{(14.371)}=3.482\right.$, $\left.p<.001, \eta_{\mathrm{p}}{ }^{2}=.127\right)$, psicoticismo $\left(F_{(14.371)}=1.991, p<.05\right.$, $\left.\eta_{\mathrm{p}}{ }^{2}=.079\right)$, el PST $\left(F_{(14.371)}=2.691, p<.01, \eta_{\mathrm{p}}{ }^{2}=.122\right)$, el GSI $\left(F_{(14.371)}=3.097, p<.001, \eta_{\mathrm{p}}{ }^{2}=.124\right)$ y el PSDI $\left(F_{(14.371)}=2.765, p<.05, \eta_{\mathrm{p}}^{2=} .097\right)$; al igual que en las EA de expresión emocional $\left(F_{(14.371)}=2.129, p<.01\right.$, 
$\left.\eta_{\mathrm{p}}^{2}=.076\right)$, pensamiento desiderativo $\left(F_{(14.371)}=1.886\right.$, $\left.p<.05, \eta_{\mathrm{p}}^{2}=.074\right)$, apoyo social $\left(F_{(14.371)}=2.955, p<.001\right.$, $\left.\eta_{\mathrm{p}}{ }^{2}=.107\right)$ y reestructuración cognitiva $\left(F_{(14.371)}=2.134\right.$, $p<.05, \eta_{\mathrm{p}}^{2}=.079$ ).

$\mathrm{Al}$ respecto, cabe destacar que los participantes que cursan carreras en el área de las ciencias de la salud y de las humanidades presentan medias más altas en el total de respuestas positivas y en el índice sintomático general, mientras que, por otra parte, la muestra perteneciente al área de las ciencias jurídicas y sociales, y ciencias de la salud presentó medias más altas en la intensidad sintomática. Con respecto a los TE, estos fueron de moderados a grandes tanto en la sintomatología psicopatológica como en las EA al estrés, destacándose los síntomas de somatización y obsesión-compulsión.

Asimismo, en cuanto a la valoración global del impacto producido por el aluvión en los participantes (donde $0=N o$ impacto/impacto mínimo y 3 = Impacto mayor o severo), se encontraron diferencias significativas en las medias de todas las dimensiones del SCL-90-R, a excepción de sensibilidad interpersonal $\left(F_{(2.371)}=2.948, p=.054\right)$. Igualmente, en este caso el TE fue pequeño, y se presentaron medias desiguales en las EA de resolución de problemas, $\left(F_{(2.371)}=11.886, p<.001, \eta_{\mathrm{p}}{ }^{2}=.061\right)$, expresión emocional $\left(F_{(2.371)}=3.825, p<.05, \eta_{\mathrm{p}}{ }^{2}=.018\right)$ y pensamiento desiderativo $\left(F_{(2.371)}=6.939, p<.01, \eta_{\mathrm{p}}^{2}=.037\right)$. Siendo más altos los valores medios alcanzados por quienes que valoran globalmente el impacto como mayor o severo.

Con respecto a la presencia de algún familiar adulto mayor o anciano, no se encontraron diferencias significativas en la sintomatología psicopatológica. Sin embargo, se observaron medias desiguales en la EA al estrés de resolución de problemas $\left(t_{(395)}=2.786, p=.006,95 \%\right.$ IC [.473, 2.741]), siendo la media más elevada en los participantes que tenían a algún anciano en su núcleo familiar. De igual forma, los universitarios que no tienen una persona mayor en su familia puntuaron más alto en evitación de problemas $\left(t_{(396)}=-2.052, p=.041,95 \%\right.$ IC $\left.[-1.875,-.040]\right)$, donde el TE fue pequeño.

Adicionalmente, con respecto a la presencia de niños en el núcleo familiar, se presentan diferencias significativas de medias en la sintomatología asociada con la somatización $\left(t_{(396)}=-2.369, p=.018,95 \%\right.$ IC[-.378, -.035]), la depresión, $\left(t_{(396)}=-2.485, p=.018,95 \%\right.$ IC[-.377, $-.044])$, la ansiedad $\left(t_{(396)}=-3.014, p=.003,95 \%\right.$ IC[-.419, $-.088])$, la ansiedad fóbica $\left(t_{(396)}=-2.695, p=.007,95 \%\right.$ IC[-.317,-.050]), el psicoticismo $\left(t_{(396)}=-2.311, p=.021\right.$, $95 \%$ IC[-.281, -.023]) y el GSI $\left(t_{(396)}=-2.418, p=.016\right.$, $95 \%$ IC[-.301,-.0310]). Cabe destacar que las medias más elevadas se presentaron en las personas que pertenecen a un núcleo familiar sin niños. Sin embargo, con respecto a las EF, no se presentan diferencias de medias a partir de la presencia de niños en la familia, a excepción de la retirada $\operatorname{social}\left(t_{(396)}=-2.445, p=.015,95 \%\right.$ IC[-2.086, -.226]), donde se encontraron puntuaciones medias superiores en las personas que habitan con familias con niños; sin embargo, acá el TE fue también pequeño.

Por otra parte, no se encontraron diferencias significativas en la sintomatología psicopatológica ni en las EA al estrés según la presencia de personas con discapacidad en el núcleo familiar. Aunque con respecto a la exposición a situaciones de violencia como robos, saqueos o disparos, sí se presentaron diferencias significativas en todos los síntomas psicopatológicos e índices globales, a excepción $\operatorname{del} \operatorname{PSDI}\left(\mathrm{t}_{(389)}=1.154, p=.249,95 \%\right.$ IC[-.056, .217]), que es un indicador de la intensidad sintomática media. Cabe destacar que las puntuaciones medias elevadas se presentan en personas que estuvieron expuestas a las situaciones de violencia, que no se presentaron medias desiguales en las EA al estrés, y que el TE fue de pequeño a moderado.

Con relación a la percepción de los participantes sobre el deterioro de la vivienda, se presentaron diferencias significativas en las medias de los síntomas psicopatológicos (TE pequeño), a excepción del índice global de intensidad sintomática $\left(\mathrm{F}_{(2.388)}=1.040, p=.073\right)$. Las medias más altas de las puntuaciones las presentan los participantes que perciben un "impacto moderado" del daño a la vivienda. Y, en cuanto a las estrategias de afrontamiento, se presentaron medias desiguales en resolución de problemas $\left(F_{(2.395)}=8.993, p<.001, \eta_{\mathrm{p}}{ }^{2}=.041\right)$, autocrítica $\left(F_{(2396)}=4.498, p<.05, \eta_{\mathrm{p}}{ }^{2}=.023\right)$, pensamiento desiderativo $\left(F_{(2.396)}=8.848, p<.001, \eta_{p}{ }^{2}=.038\right)$, y reestructuración cognitiva $\left(F_{(2.396)}=4.015, p<.05, \eta_{\mathrm{p}}{ }^{2}=.017\right)$, donde las puntuaciones medias más elevadas se presentaron en quienes perciben un "impacto mayor o severo" en del deterioro de la vivienda.

También, con respecto al acceso a servicios básicos después al aluvión se presentaron diferencias de medias en la categoría de ausencia de "agua potable" $(0=$ No ausencia hasta $4=$ Más de 15 días), siendo los más significativos en los síntomas psicopatológicos de somatización $\left(F_{(4.383)}=4.694\right.$, $p<.001)$, obsesión-compulsión $\left(F_{(4.383)}=3.742, p<.01\right)$, depresión $\left(F_{(4.383)}=3.268, p<.01\right)$, ansiedad $\left(F_{(4.383)}=2.297\right.$, $p<.01)$, ansiedad fóbica $\left(F_{(4.383)}=4.412, p<.01\right)$ y psicoticismo $\left(F_{(4.383)}=2.946, p<.05\right)$, y en los índices GSI $\left(F_{(4.383)}=3.499, p<.01\right)$ y PSDI $\left(F_{(4.377)}=3.320, p<.01\right)$. Acá, las puntuaciones medias más altas correspondieron a la categoría de "Más de 15 días" en ausencia de agua potable; y con respecto a las EA al estrés, las diferencias se presentaron en autocrítica $\left(F_{(4.382)}=2.595, p<.05\right)$, expresión emocional $\left(F_{(4.383)}=2.986, p<.05\right)$, pensamiento 
desiderativo $\left(F_{(4.383)}=2.557, p<.05\right)$ y retirada social $\left(F_{(4.383)}=4.818, p<.001\right)$. Acá, las medias más altas se presentaron en los participantes que estuvieron más de siete días en ausencia del servicio, y los TE fueron pequeños.

Por último, en cuanto a la situación laboral o pérdida de ingresos, se presentaron medias desiguales en casi todos los síntomas psicopatológicos, excepto en sensibilidad interpersonal $\left(t_{(387)}=1.371, p=.171\right)$, ideación paranoide $\left(t_{(387)}=1.912, p=.057\right)$ y psicoticismo $\left(t_{(387)}=1.875\right.$, $p=.062$ ). Y acá, con respecto a las EA al estrés, se observaron diferencias significativas de medias en autocrítica $\left(t_{(387)}=3.226, p<.01,95 \% \mathrm{IC}[.439,1.767]\right)$, pensamiento desiderativo $\left(t_{(387)}=3.154, p<.01,95 \% \mathrm{IC}[.689,2.968]\right)$, evitación de problemas $\left(t_{(387)}=3.696, p<.001,95 \%\right.$ IC $[.852,2.788])$ y retirada social $\left(t_{(387)}=4.498, p<.001\right.$, $95 \%$ IC[1.214, 3.100]). También, las medias más altas en las puntuaciones obtenidas tanto en el SCL-90-R como en el CSI correspondieron a las personas insertas en un núcleo familiar afectado laboral y económicamente.

\section{Correlaciones entre síntomas psicopatológicos y EA al estrés}

Con el fin de explorar la relación entre los síntomas psicopatológicos de los universitarios y las EA al estrés, se realizaron correlaciones entre cada una de las dimensiones, y tal como se observa en la Tabla 2, la correlación entre las dimensiones del SCL-90-R y el CSI es baja y en algunos casos moderada. Específicamente, las estrategias de apoyo social y reestructuración cognitiva no correlacionaron con los síntomas psicopatológicos ni con los índices globales, y la evitación de problemas solo presentó correlaciones significativas con los síntomas de ideación paranoide y psicoticismo. Sin embargo, la estrategia de retirada social correlacionó significativamente con todos los síntomas psicopatológicos de depresión $\left(r_{(398)}=.436, p=.001\right)$, ansiedad $\left(r_{(398)}=.415, p=.001\right)$ y con el GSI $\left(r_{(398)}=.438, p=.001\right)$.

Así mismo, la estrategia de pensamiento desiderativo correlacionó significativamente con todas las dimensiones psicopatológicas y los índices globales, donde destacan las correlaciones con obsesión-compulsión $\left(r_{(398)}=.335\right.$, $p=.001)$, depresión $\left(r_{(398)}=.311, p=.001\right)$, somatización $\left(r_{(398)}=.309, p=.001\right)$, el GSI $\left(r_{(398)}=.320, p=.001\right) \mathrm{y} \mathrm{el}$ $\operatorname{PST}\left(r_{(398)}=.328, p=.001\right)$. Acá, cabe destacar que aunque se presentaron correlaciones significativas $(p<.05, p<.01)$, estas son moderadas, puesto que tienen coeficientes menores a .4 (Aron \& Aron, 2002).

Variables predictoras de las dimensiones psicopatológicas e indices globales

Para explorar la potencia predictiva de las EA al estrés, el sexo, la edad y las variables relacionadas con la percepción sobre la experiencia de vida durante y después al aluvión sobre las variables criterio de los síntomas psicopatológicos y los índices globales se realizó un análisis de regresión lineal múltiple con el método por pasos (stepwise).

Tabla 2

Matriz de correlaciones de Pearson entre EA al estrés y sintomatología psicopatológica

\begin{tabular}{lcccccccc}
\hline \multirow{2}{*}{ SCL-90-R } & \multicolumn{7}{c}{ Estrategias de Afrontamiento al Estrés (CSI) } \\
\cline { 2 - 7 } & REP & AUT & EEM & PSD & APS & REC & EVP & RES \\
\hline Total síntomas positivos (PST) & $.120^{*}$ & $.194^{* *}$ & $.111^{*}$ & $.328^{* *}$ & -.004 & -.015 & .058 & $.361^{* *}$ \\
Índice sintomático general (GSI) & $.145^{* *}$ & $.194^{* *}$ & $.132^{* *}$ & $.320^{* *}$ & -.035 & .001 & .066 & $.438^{* *}$ \\
Intensidad sintomática (PSDI) & $.172^{* *}$ & $.100^{*}$ & $.114^{* *}$ & $.193^{* *}$ & -.032 & .088 & .096 & $.332^{* *}$ \\
Somatización & $.179 *$ & $.187^{* *}$ & .092 & $.309^{* *}$ & -.053 & -.003 & .000 & $.353^{* *}$ \\
Obsesión-compulsión & $.204^{* *}$ & $.162^{* *}$ & $.176^{* *}$ & $.335^{* *}$ & .082 & .096 & .008 & $.368^{* *}$ \\
Sensibilidad interpersonal & .075 & $.147^{* *}$ & $.144^{* *}$ & $.269^{* *}$ & .003 & -.029 & .067 & $.319^{* *}$ \\
Depresión & $.130^{*}$ & $.167^{* *}$ & $.160^{* *}$ & $.311^{* *}$ & -.036 & -.022 & .008 & $.436^{* *}$ \\
Ansiedad & .087 & $.208^{* *}$ & .097 & $.281^{* *}$ & -.096 & -.052 & .085 & $.415^{* *}$ \\
Hostilidad & .081 & .094 & .040 & $.248^{* *}$ & -.065 & -.048 & .067 & $.332^{* *}$ \\
Ansiedad fóbica & .053 & $.160^{* *}$ & $.114^{*}$ & $.228^{* *}$ & -.062 & .035 & .051 & $.338^{* *}$ \\
Ideación paranoide & $.164 * *$ & $.206^{* *}$ & $.112^{*}$ & $.245^{* *}$ & .009 & .048 & $.155^{* *}$ & $.395^{* *}$ \\
Psicoticismo & .050 & $.175^{* *}$ & .079 & $.198^{* *}$ & -.037 & -.013 & $.140^{* *}$ & $.379^{* *}$ \\
\hline
\end{tabular}

Nota. REP: Resolución de problemas, AUT: Autocrítica, EEM: Expresión emocional, PSD: Pensamiento desiderativo, APS: Apoyo social, REC: Restructuración cognitiva, EVP: Evitación de problemas, RES: Retirada social. *p $<.05, * * p<.01$. 
Tabla 3

Resumen del modelo de regresión por pasos en la sintomatología psicopatológica

\begin{tabular}{|c|c|c|c|c|c|}
\hline Dimensiones SCL-90-R & Variables independientes & Beta & $\mathrm{t}$ & $\mathrm{F}$ & R2 Corregida \\
\hline \multirow{5}{*}{ Índice sintomático general (GSI) } & Retirada social & .365 & $7.872 * * *$ & \multirow{5}{*}{$28.892 * * *$} & \multirow{5}{*}{.286} \\
\hline & Pensamiento desiderativo & .224 & $4.762 * * *$ & & \\
\hline & Exposición a la violencia & -.127 & $-2.780 * *$ & & \\
\hline & Valoración de impacto & .128 & $2.767 * *$ & & \\
\hline & Sexo & .121 & $2.623 * *$ & & \\
\hline \multirow{6}{*}{ Total de sintomas positivos (PST) } & Retirada social & .255 & $5.158 * * *$ & \multirow{6}{*}{$20.229 * * *$} & \multirow{6}{*}{.248} \\
\hline & Pensamiento desiderativo & .311 & $6.246 * * *$ & & \\
\hline & Deterioro de vivienda & .106 & $2.140 * *$ & & \\
\hline & Reestructuración cognitiva & -.134 & $-2.765^{* *}$ & & \\
\hline & Autocrítica & .118 & $2.362 * *$ & & \\
\hline & Exposición a la violencia & -.104 & $-2.173 * *$ & & \\
\hline \multirow{4}{*}{ Intensidad sintomática (PSDI) } & Retirada social & .282 & $5.412 * * *$ & \multirow{4}{*}{$14.109 * * *$} & \multirow{4}{*}{.133} \\
\hline & Sexo & .148 & $2.927 * *$ & & \\
\hline & Valoración de impacto & .133 & $2.622 * *$ & & \\
\hline & Situación laboral & -.103 & $-1.997 *$ & & \\
\hline \multirow{7}{*}{ Somatización } & Retirada social & .225 & $4.487 * * *$ & \multirow{7}{*}{$19.099 * * *$} & \multirow{7}{*}{.266} \\
\hline & Pensamiento desiderativo & .268 & $5.207 * * *$ & & \\
\hline & Sexo & .159 & $3.380 * *$ & & \\
\hline & Valoración de impacto & .151 & $3.208 * *$ & & \\
\hline & Exposición a violencia & -.158 & $-3.401 * *$ & & \\
\hline & Apoyo social & -.127 & $-2.509^{*}$ & & \\
\hline & Autocrítica & .112 & $2.251^{*}$ & & \\
\hline \multirow{4}{*}{ Obsesión-compulsión } & Retirada social & .290 & $6.171 * * *$ & \multirow{4}{*}{$31.567 * * *$} & \multirow{4}{*}{.259} \\
\hline & Pensamiento desiderativo & .269 & $5.680 * * *$ & & \\
\hline & Exposición a la violencia & -.190 & $-4.092 * * *$ & & \\
\hline & Valoración de impacto & .112 & $2.383^{*}$ & & \\
\hline \multirow{6}{*}{ Sensibilidad interpersonal } & Retirada social & .237 & $4.793 * * *$ & \multirow{6}{*}{$13.314 * * *$} & \multirow{6}{*}{.175} \\
\hline & Pensamiento desiderativo & .193 & $3.432 * *$ & & \\
\hline & Exposición a la violencia & -.084 & $-1.652 *$ & & \\
\hline & Reestructuración cognitiva & -.145 & $-2.813^{* *}$ & & \\
\hline & Expresión emocional & .143 & $2.591^{*}$ & & \\
\hline & Deterioro de la vivienda & .105 & $2.049 *$ & & \\
\hline \multirow{6}{*}{ Depresión } & Retirada social & .395 & $8.625 * * *$ & \multirow{6}{*}{$25.180 * * *$} & \multirow{6}{*}{.294} \\
\hline & Pensamiento desiderativo & .198 & $3.782 * * *$ & & \\
\hline & Sexo & .141 & $3.062 * *$ & & \\
\hline & Reestructuración cognitiva & .119 & $2.568^{*}$ & & \\
\hline & Deterioro de la vivienda & -.134 & $-2.805 * *$ & & \\
\hline & Expresión emocional & .122 & $2.393 *$ & & \\
\hline \multirow{8}{*}{ Ansiedad } & Retirada social & .272 & $5.428 * * *$ & \multirow{8}{*}{$16.886 * * *$} & \\
\hline & Pensamiento desiderativo & .255 & $4.940 * * *$ & & \\
\hline & Sexo & .124 & $2.638 * *$ & & \\
\hline & Valoración de impacto & .111 & $2.367 *$ & & \\
\hline & Presencia de niños & .106 & $2.297 *$ & & .267 \\
\hline & Autocrítica & .127 & $2.547 *$ & & \\
\hline & Exposición a violencia & -.108 & $-2.313^{*}$ & & \\
\hline & Apoyo social & -.143 & $-2.832 * *$ & & \\
\hline
\end{tabular}


Continuación tabla 3

\begin{tabular}{|c|c|c|c|c|c|}
\hline Dimensiones SCL-90-R & Variables independientes & Beta & $\mathrm{t}$ & $\mathrm{F}$ & R2 Corregida \\
\hline \multirow{6}{*}{ Hostilidad } & Retirada social & .256 & $5.071^{* * *}$ & \multirow{6}{*}{$13.014 * * *$} & \multirow{6}{*}{.171} \\
\hline & Pensamiento desiderativo & .205 & $3.942 * * *$ & & \\
\hline & Reestructuración cognitiva & -.141 & $-2.774 * *$ & & \\
\hline & Deterioro de vivienda & .147 & $2.756^{* *}$ & & \\
\hline & Ausencia de electricidad & -.123 & $-2.371 *$ & & \\
\hline & Situación laboral & -.108 & $-2.067^{*}$ & & \\
\hline \multirow{5}{*}{ Ansiedad fóbica } & Retirada social & .280 & $5.643 * * *$ & \multirow{5}{*}{$16.410 * * *$} & \multirow{5}{*}{.181} \\
\hline & Pensamiento desiderativo & .177 & $3.563^{* * *}$ & & \\
\hline & Sexo & .145 & $2.944 * *$ & & \\
\hline & Exposición a violencia & -.119 & $-2.423 *$ & & \\
\hline & Presencia de niños & .107 & $2.187^{*}$ & & \\
\hline \multirow{3}{*}{ Ideación paranoide } & Retirada social & .339 & $6.637 * * *$ & \multirow{3}{*}{$28.587 * * *$} & \multirow{3}{*}{.192} \\
\hline & Pensamiento desiderativo & .185 & $3.786^{* * *}$ & & \\
\hline & Autocrítica & .106 & $2.108 *$ & & \\
\hline \multirow{4}{*}{ Psicoticismo } & Retirada social & .334 & $6.749^{* * *}$ & \multirow{4}{*}{$19.301 * * *$} & \multirow{4}{*}{.173} \\
\hline & Deterioro de vivienda & .131 & $2.632 * *$ & & \\
\hline & Pensamiento desiderativo & .112 & $2.224^{*}$ & & \\
\hline & Presencia de niños & .098 & $1.996^{*}$ & & \\
\hline
\end{tabular}

Nota. $* * * p<.001, * * p<.01, * p<.05$.

En la Tabla 3 se pueden observar los predictores que entraron en el último paso en la ecuación de regresión para cada dimensión psicopatológica y los índices globales. Con respecto al GSI y PST, estos se predijeron principalmente por la retirada social $\left(F_{(5.344)}=28.892, p<.001\right) \mathrm{y}$ el pensamiento desiderativo $\left(F_{(6.344)}=20.229, p<.001\right)$. Por otra parte, la retirada social fue un predictor significativo para el PSDI, junto al sexo y la valoración de impacto $\left(F_{(4.339)}=14.109, p<.001\right)$.

Además, con relación a la sintomatología psicopatológica, los principales predictores para la somatización fueron retirada social y pensamiento desiderativo $\left(\mathrm{F}_{(7.342)}=19.099\right.$, $p<.001$ ); mientras que la retirada social, el pensamiento desiderativo y la exposición a la violencia fueron predictores significativos para la obsesión-compulsión $\left(F_{(4.345)}=31.567, p<.001\right)$; así como la sensibilidad interpersonal, la depresión, la ansiedad, la hostilidad, la ansiedad fóbica y la ideación paranoide fueron predichas principalmente por la retirada social y el pensamiento desiderativo $\left(F_{(6.343)}=13.314, p<.001 ; F_{(6.343)}=25.810\right.$, $p<.001 ; F_{(8.341)}=16.886, p<.001 ; F_{(6.343)}=13.014, p<.001$; $F_{(5.344)}=28.892, p<.001 ; F_{(5.344)} \stackrel{(6.34)}{=} 6.410, p<.001 ; \mathrm{y}$ $F_{(3.346)}=28.587, p<.001$, respectivamente); y la retirada social fue un buen predictor, junto al deterioro de vivienda para el psicoticismo $\left(F_{(4.345)}=19.301, p<.001\right)$.

Por último, las dimensiones psicopatológicas más explicadas por las variables predictoras fueron depresión
(29.4\%), el índice sintomático general (28.6\%), la ansiedad (26.7\%), la somatización (26.6\%), la obsesión-compulsión $(25.9 \%)$ y el total de sintomas positivos $(24.8 \%)$.

\section{DISCUSIÓN}

El presente estudio tuvo como objetivo identificar la asociación entre la sintomatología psicopatológica, las EA y las variables sociodemográficas relacionadas con la vivencia del desastre socionatural de aluvión de barro en una ciudad de tamaño mediano.

Como resultado se encuentra la existencia de diferencias debidas al sexo en los síntomas psicopatológicos de somatización, depresión y ansiedad, y las EA al estrés, lo cual concuerda con lo planteado por Kelly, Tyrka, Price y Carpente (2008) dada la tendencia en las mujeres a experimentar mayor ansiedad y a la escogencia de la EA uso de las emociones. Del mismo modo, los resultados obtenidos son congruentes con lo evidenciado por Novais et al. (2017), puesto que estos también señalan que el sexo femenino es más proclive al desarrollo de sintomatología ansiosodepresiva, dada una mayor activación neurofisiológica e inmunológica en este. No obstante, existe evidencia de que en situaciones de estrés crónico o intenso, esta diferencia no ocurre (Matud, 2004; Baker, Kentner, Konkle, SantaMaria \& Bielajew, 2006). 
Del mismo modo se encontraron diferencias entre las EA al estrés y sexo, puesto que las mujeres puntuaron más alto en expresión emocional y pensamiento desiderativo; así como los hombres lo hicieron en evitación del problema; hallazgo en concordancia con las conclusiones de estudios meta-analíticos previos (Meléndez, Mayordomo, Sancho \& Tomas, 2012).

Con respecto a las EA ante estresores extremos, a diferencia de lo planteado por Coiro, Bettis y Compas (2017), donde no hubo mediación de las EA ante situaciones estresantes de un carácter más habitual como el estrés académico, en el presente estudio sí se observan diferencias entre las EA y la sintomatología. Esto confirma la evidencia en relación a la respuesta conductual ante los estresores percibidos como más controlables, a diferencia de estresores percibidos como menos controlables-como aquellos que aparecen ante los desastres socionaturales-que conllevan conjuntamente sintomatología explicita y un deterioro en la competencia social (Clarke, 2006).

Asimismo, en los resultados se observa el potencial predictivo de las variables sociodemográficas relacionadas con la experiencia de vivencia del desastre socionatural y las EA al estrés, donde se detectó, sobre todo, la presencia de: (a) niños, ancianos o personas con discapacidad en el núcleo familiar, (b) la exposición a violencia, (c) el deterioro de la vivienda, y (d) la valoración de impacto en servicios básicos o perdida del trabajo sobre los síntomas psicopatológicos (depresión, indice sintomático general, ansiedad, somatización, obsesión-compulsión y el total de sintomas positivos).

De este modo, al considerar el papel de la familia en la modulación de la respuesta de estrés ante situaciones de desastres, se abren futuros temas de investigación para el estudio de las EA, sobre todo al tener en cuenta que las familias no presentan un estilo único de afrontamiento, sino diferentes estrategias dependiendo de las demandas que surgen a lo largo del proceso de adaptación a una experiencia de estrés extremo, lo que podría representar tanto un factor de vulnerabilidad como uno protector, por su capacidad de movilizar recursos (Macías, Madariaga, Valle \& Zambrano, 2013).

Por otra parte, las diferencias con respecto a la valoración global del impacto producido por el aluvión en todas las dimensiones psicopatológicas, a excepción de sensibilidad interpersonal, confirman la multiplicidad de efectos que posee la experiencia del estrés. Estudios previos han constatado que en la población universitaria afectada por un aluvión de barro, un gran porcentaje de jóvenes expuestos a una situación de catástrofe manifiestan un alto impacto subjetivo (Lería \& Salgado, 2016).

Finalmente, este estudio provee evidencia empírica para el análisis de las conductas adaptativas ante desastres socionaturales en población adulta-joven de estudiantes universitarios, y se constituye en un aporte a las intervenciones terapéuticas para el desarrollo de las EA al estrés en un sentido preventivo. Por otra parte, sus limitaciones apuntan principalmente al carácter transeccional y no longitudinal del diseño, lo que no permite visualizar la progresión en la aparición de la sintomatología psicopatológica y la asociación con las EA. No obstante, las proyecciones de este estudio podrían orientar la investigación sobre otros sectores de la población que fueron afectados directa e indirectamente por el aluvión, tanto individual como grupalmente, y donde se integren otras variables como, por ejemplo, el bienestar subjetivo y la satisfacción vital.

\section{REFERENCIAS}

Amón, J. (1996). Estadística para Psicólogos II. Probabilidad. Estadística Inferencial ( $9^{\mathrm{a}}$ ed.). Ediciones Pirámide: Madrid, España.

Aledi, A., \& Sulaiman, S. (2014). La incuestionabilidad del riesgo. Ambiente \& Sociedade, 17(4),9-16. DOI: https:// dx.doi.org/10.1590/1809-4422ASOCEx01V1742014.

Amón, J. (1995). Estadística para Psicólogos I. Probabilidad. Estadística Descriptiva (15 a ed.). Madrid, España: Ediciones Pirámide.

Amón, J. (1996). Estadística para Psicólogos II. Probabilidad. Estadística Inferencial (9 $9^{\mathrm{a}}$ ed.). Madrid, España: Ediciones Pirámide.

Armitage, P., Berry, G., \& Matthews, J. (1994). Statistical methods in medical research ( $3^{\mathrm{a}}$ ed.). Oxford, Reino Unido: Blackwell Science.

Aron, A., \& Aron, E. (2002). Estadística para Psicología (2a ed.). Buenos Aires, Argentina: Editorial Prentice Hall.

Ato, M., López, J., \& Benavente, A. (2013). Un sistema de clasificación de los diseños de investigación en psicología. Anales de Psicología, 29(3), 1038-1059. doi:10.6018/analesps.29.3.178511.

Baker, S. L., Kentner, A. C., Konkle, A. T., Santa-Maria Barbagallo, L., \& Bielajew, C. (2006). Behavioral and physiological effects of chronic mild stress in female rats. Physiology \& Behavior, 87(2), 314-322. DOI: http://dx.doi. org/10.1016/j.physbeh.2005.10.019.

Balaban, V. (2006). Psychological assessment of children in disasters and emergencies. Disasters, 30(2),178-198. doi:10.1111/j.0361-3666.2006.00314.x.

Becker, K. A., Turner, H. A., \& Finkelhor, D. (2010). Disasters, Victimization, and Children's Mental Health. Child Development, 81(4), 1040-1052. doi:10.1111/j.14678624.2010.01453.x.

Blaustein, J. D., \& Ismail, N. (2013). Enduring influence of pubertal stressors on behavioral response to hormones in female mice. Horones and Behavior, 64(2), 390-398. DOI: http://dx.doi.org/10.1016/j.yhbeh.2013.01.015. 
Cano, G. F., Rodríguez, F. L., \& García, M. J. (2007). Spanish version of the Coping Strategies Inventory. Actas españolas de psiquiatría, 35(1), 29-39. Recuperado de http://personal. us.es/fjcano/drupal/files/AEDP\%2007\%20(esp).pdf.

Clarke, S. E., \& Chenoweth, E. (2006). The politics of vulnerability: Constructing local performance regimes for homeland security. Review of Policy Research, 23(1), 95-114. DOI: http://dx.doi.org/10.1111/j.1541-1338.2006.00187.x.

Cohen, J. (1988). Statistical power analysis for the behavioral sciences (2nd ed.). Hillsdale, NJ: Lawrence Earlbaum Associates.

Coiro, M. J., Bettis, A. H., \& Compas, B. E. (2017). College students coping with interpersonal stress: Examining a control-based model of coping. Journal of American College Health, 65(3),177-186. doi:10.1080/07448481.2016.12666 41.

Craparo, G., Faraci, P., Rotondo, G., \& Gori, A. (2013). The Impact of Event Scale - Revised: psychometric properties of the Italian version in a sample of flood victims. Neuropsychiatric Disease and Treatment, 9, 1427-1432. doi:10.2147/ NDT.S51793.

Daxing, W., Huifang, Y., Shujing, X., \& Ying, Z. (2011). Risk factors for posttraumatic stress reactions among Chinese students following exposure to a snowstorm disaster. BMC Public Health, 11(96), 1-7. DOI: http://dx.doi. org/10.1186/1471-2458-11-96.

Derogatis, L. R. (2002). SCL-90-R Symptom Checklist 90 Revised (Manual). Madrid: TEA Ediciones.

González, J. L., De las Cuevas, C, Rodríguez, M., \& Rodríguez, F. (2002). SCE-90-R, Symptom Checklist 90 Revised, Spanish adaptation. Madrid: TEA.

Gunnar, M. R., Wewerka, S., Frenn, K., Long, J. D., \& Griggs, C. (2009). Developmental changes in hypothalamus-pituitary-adrenal activity over the transition to adolescence: normative changes and associations with puberty. Development and Psychopathology, 21(1), 69-85. http://dx.doi. org/10.1017/S0954579409000054.

Hussain, A., Weisæth, L., \& Heir, T. (2013). Posttraumatic stress and symptom improvement in Norwegian tourists exposed to the 2004 tsunami - a longitudinal study. BMC Psychiatry, 13(232), 1-11. doi:10.1186/1471-244X-13-232.

Kelly, M. K., Tyrka, A. R., Price, L. H., \& Carpenter, L. L. (2008). Sex differences in the use of coping strategies: predictors of anxiety and depressive symptoms. Depression and Anxiety, 25(10), 839-846. doi:10.1002/da.20341.

Lazarus, R. S., \& Folkman, S. (1986). Estrés y procesos cognitivos (2 $2^{\mathrm{a}}$. ed.). Madrid: Martínez Roca.

Leach, J. (2016). Psychological factors in exceptional, extreme and torturous environments. Extrememe Physiology \& Medicine, 5, 7. DOI 10.1186/s13728-016-0048-y.

Leiva-Bianchi, M. (2011). Relevancia y prevalencia del estrés post-traumático post-terremoto como problema de salud pública en Constitución, Chile. Revista de Salud pública
13(4), 551-559. Recuperado de http://www.scielosp.org/ pdf/rsap/v13n4/v13n4a01.pdf.

Lería Dulčić, F., \& Salgado Roa, J. (2016). Estrés posttraumático y estrés subjetivo en estudiantes universitarios tras aluvión de barro. Ciencias Psicológicas, 10(2), 129141. doi:dx.doi.org/10.22235/cp.v10i2.1250.

Lima, B. R., Santacruz, H., Lozano, J., Luna, J., \& Pai S. (1988). Primary mental health care for the victims of the disaster in Armero, Colombia. Acta Psiquiátrica y Psicológica de América Latina, 34(1),13-32.

Ling-Xiang, X., \& Cody, D. (2011). The Relationship between Interpersonal Traits and Posttraumatic Stress Disorder Symptoms: Analyses from Wenchuan Earthquake Adolescent Survivors in China. Journal of Traumatic Stress, 24(4), 487-490. doi:10.1002/jts.20655.

Loo, G. T., DiMaggio, C. J., Gershon, R. R., Canton, D. B., Morse, S. S., \& Galea, S. (2016). Coping Behavior and Risk of Post-Traumatic Stress Disorder Among Federal Disaster Responders. Disaster Medicine and Public Health Preparedness, 10(1),108-17. doi:10.1017/dmp.2015.141.

López, J., Christodoulou, G., Maj, M., Sartorius, N., \& Okasha, A. (2005). Disasters and Mental Health. The Atrium, Southern Gate, Chichester, England: WILEY.

Macías, M. A., Madariaga, C., Valle, M. A., \& Zambrano, J. (2013). Estrategias de afrontamiento individual y familiar frente a situaciones de estrés psicológico. Psicología desde el Caribe, 30(1), 123-145. Recuperado de http://www.redalyc.org/pdf/213/21328600007.pdf.

Matud Aznar, M. (2004). Impacto de la violencia doméstica en la salud de la mujer maltratada. Psicothema, 16(3), 397401. Recuperado de http://www.psicothema.com/pdf/3009. pdf.

Meléndez, J. C., Mayordomo, T., Sancho P., \& Tomás, J. M. (2012). Coping strategies: gender differences and development throughout life span. The Spanish Journal of Psychology, 15(3),1089-98. DOI: http://dx.doi.org/10.5209/ rev_SJOP.2012.v15.n3.39399.

Mestre, V., Samper, P., Tur-Porcar, A. M., Richaud de Minzi, M. C., \& Mesurado, B. (2012). Emociones, estilos de afrontamiento y agresividad en la adolescencia. Universitas Psychologica, 11, 1263-1275. Recuperado de http://revistas.javeriana.edu.co/index.php/revPsycho/article/download/1831/3356.

Ministerio de Vivienda y Urbanismo (2015). MINVU presenta estrategia para reconstrucción de la región de Atacama. Recuperado de http://www.minvu.cl/opensite det_20150422130517.aspx.

Momma, H., Niu, K., Kobayashi, Y., Huang, C., Otomo, A., Chujo, M., ... Nagatomi, R. (2014). Leg Extension Power Is a Pre-Disaster Modifiable Risk Factor for Post-Traumatic Stress Disorder among Survivors of the Great East Japan Earthquake: A Retrospective Cohort Study. PLOS ONE, 9(4), 1-10. doi: http://dx.doi.org/10.1371/journal. pone. 0096131 . 
Montgomery, C., Trumpower, D., McMurtry, A., Ghani, S., Daubney, A., \& Guerin, E. (2014). Adolescent Stress and Coping: A Meta-analysis. Ontario Health Promotion E-Bulletin, 849. Recuperado de http://webcache.googleusercontent.com/search?q=cache:oJouSYVEPKwJ:https://www. ohpe.ca/node $/ 15588 \&$ num $=1 \& \mathrm{hl}=\mathrm{en} \& \mathrm{gl}=\mathrm{cl} \& \mathrm{strip}=1 \& \mathrm{vw}$ $\mathrm{src}=0$.

Morales, F. M., Trianes, M. V., Miranda, J., \& Inglés, C. J. (2016). Prevalence of strategies for coping with daily stress in children. Psicothema, 28(4), 370-376. Recuperado de http://www.redalyc.org/pdf/727/72747804002.pdf.

Neria, Y., Nandi, A., \& Galea, S. (2008). Post-traumatic stress disorder following disasters: A systematic review. Psychological Medicine, 38(4), 467-480. doi: 10.1017/ S0033291707001353.

Novais, A., Monteiro, S., Roque, S., Correia-Neves, M., \& Sousa, N. (2017). How age, sex and genotype shape the stress response. Neurobiology of Stress, 6, 44-56. http://dx.doi. org/10.1016/j.ynstr.2016.11.00.

Ortiz, C. D., Wendy, K., Silverman, J., Jaccard, J., \& La Greca, A. M. (2011). Children's State Anxiety in Reaction to Disaster Media Cues: A Preliminary Test of a Multivariate Model. Psychological Trauma: Theory, Research, Practice, and Policy, 3(2), 157-164. doi:10.1037/a0020098.

Pérez, C. I., Vicente, B., Zlotnick, C., Kohn, R., Johnson, J., Valdivia, S., \& Rioseco, P. (2009). Estudio epidemiológico de sucesos traumáticos, trastorno de estrés post-traumático y otros trastornos psiquiátricos en una muestra representativa de Chile. Salud Mental, 32(2), 145-153. Recuperado de https:/www.ncbi.nlm.nih.gov/pmc/articles/PMC2990643/ pdf/nihms160797.pdf.

Pfefferbaum, B., Nitiéma, P., Jacobs, A. K., Noffsinger, M. A., Wind, L. H., \& Allen, S. F. (2016). Review of coping in children exposed to mass trauma: measurement tools, coping styles, and clinical implications. Prehospitl and Disaster Medicine, 1(2), 169-180. doi:10.1017/ S1049023X16000169.

Pinchen, Y., Cheng-Fang, Y., Tze-Chun, T., Cheng-Sheng, C., Rei-Cheng, Y., Ming-Shyan, H., ... Hsin-Su, Y. (2011). Posttraumatic stress disorder in adolescents after Typhoon Morakot-associated mudslides. Journal of Anxiety Disorders, 25, 362-368. doi:10.1016/j.janxdis.2010.10.010.
Pineda, C., \& López, W. (2010). Atención Psicológica Postdesastres: Más que un "Guarde la Calma". Una Revisión de los Modelos de las Estrategias de Intervención. Terapia psicológica, 28(2), 155-160. https://dx.doi.org/10.4067/ S0718-48082010000200003.

Powell, T., \& Bui, T. (2016). Supporting Social and Emotional Skills After a Disaster: Findings from a Mixed Methods Study. School Mental Health, 8, 106-119. doi: 10.1007/ s12310-016-9180-5.

Roberts, Y. H., Witman, M., Mitchell, M. J., \& Taffaro, C. (2010). Mental health symptoms in youth affected by Hurricane Katrina. Professional Psychology: Research \& Practice, 41(1), 10-18. doi:10.1037/a0018339.

Rosa-Alcázar, A. I., Parada-Navas, J. L., \& Rosa-Alcázar, Á. (2014). Síntomas psicopatológicos en adolescentes españoles: relación con los estilos parentales percibidos y la autoestima. Anales de psicología, 30(1), 133-142. Recuperado de http://www.redalyc.org/pdf/167/16729452014.pdf.

Salloum, A., \& Overstreet, S. (2012). Grief and trauma intervention for children after disaster: Exploring coping skills versus trauma narration. Behaviour Research and Therapy, 50, 169-179. doi:10.1016/j.brat.2012.01.001.

Sánchez, R. O., \& Ledesma, R. D. (2009). Análisis psicométrico del Inventario de Síntomas Revisado (SCL-90-R) en población clínica. Revista Argentina De Clínica Psicológica, 18(3), 265-274. Recuperado de http://www.redalyc.org/ pdf/2819/281921775007.pdf.

Stratta, P., Capanna, C., Dell'Osso, I., Carmassi, C., Patriarca, S., Di Emidio, G., Riccardi, Collazzoni, A., \&, Rossi, A. (2015). Resilience and coping in trauma spectrum symptoms prediction: A structural equation modeling approach. Personality and Individual Differences, 77, 55-61. doi: 10.1016/j.paid.2014.12.035.

Tobin, D. L., Holroyd, K. A., Reynolds, R. V., \& Kigal, J. K. (1989). The hierarchical factor structure of the Coping Strategies Inventory. Cognitive Therapy and Research, 13(4), 343-361. doi: 10.1007/BF01173478.

Wadsworth, M. E., Raviv, T., Compas, B. E., \& Connor-Smith, J. K. (2005). Parent and adolescent responses to povertyrelated stress: Tests of mediated and moderated coping models. Journal of Child and Family Studies, 14(2), 283298. DOI: http://dx.doi.org/10.1007/s10826-005-5056. 